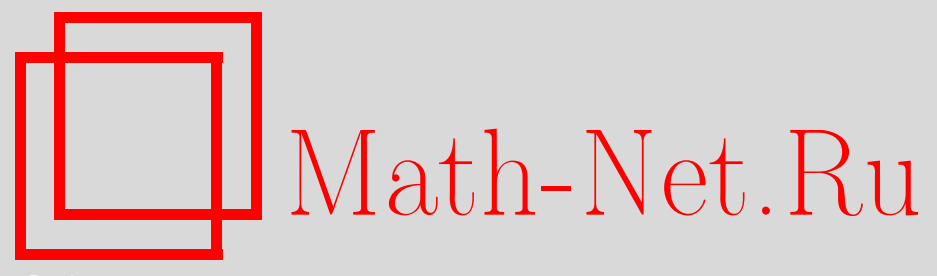

М. И. Тихомирова, В. П. Чистяков, О двух статистиках типа хи-квадрат, построенных по частотам цепочек состояний сложной цепи Маркова, Дискрет. матем., 2003, том 15, выпуск 2, 149-159

DOI: https://doi.org/10.4213/dm202

Использование Общероссийского математического портала Math-Net.Ru подразумевает, что вы прочитали и согласны с пользовательским соглашением http: //www . mathnet.ru/rus/agreement

Параметры загрузки:

IP : 52.205 .19 .152

26 апреля 2023 г., 13:20:57

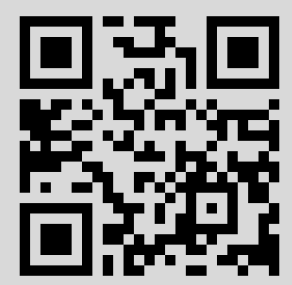




\title{
О двух статистиках типа хи-квадрат, построенных по частотам цепочек состояний сложной цепи Маркова
}

\author{
() 2003 г. М. И. Тихомирова, В. П. Чистяков
}

\begin{abstract}
Рассматривается последовательность состояний $(s-1)$-сложной цепи Маркова, У которой вероятности переходов зависят только от небольшой части $s-1$ предыдущих состояний. Получены предельные распределения $\chi^{2}$-статистик $X$ и $Y$, построенных по частотам цепочек состояний цепи Маркова. Для статистики $X$ использовались частоты цепочек только тех состояний, от которых зависят вероятности переходов, а для статистики $Y$ - частоты сплошных $s$-цепочек. Статистический критерий со статистикой $X$, различающий гипотезы $H_{1}$ (сложная цепь Маркова) и $H_{0}$ (независимая равновероятная последовательность) оказался более мощным по сравнению с аналогичным критерием со статистикой $Y$. Статистика $Z$ критерия Неймана-Пирсона так же, как и $X$, зависит только от частот несплошных цепочек. Статистики $X$ и $Y$ вычисляются без использования параметров распределения при гипотезе $H_{1}$, а их вероятности ошибок 1-ого и 2-ого рода зависят только от параметра нецентральности, являющегося функцией от вероятностей переходов. Таким образом, для этих статистик гипотезу $H_{1}$ можно рассматривать как сложную.

Работа выполнена при поддержке Российского фонда фундаментальных исследований, проект 00-15-96136.
\end{abstract}

\section{1. Сложная цепь Маркова}

При проверке качества последовательности случайных чисел одной из возможных конкурирующих гипотез по отношению к основной гипотезе $H_{0}$ о независимости и равновероятности элементов последовательности является предположение о том, что исследуемая последовательность является сложной цепью Маркова, у которой вероятности переходов близки к вероятностям равномерного распределения на состояниях, а зависимость от прошлого в переходных вероятностях ограничивается небольшим числом элементов, удаленных от данного момента на заданные расстояния.

Рассмотрим (s-1)-сложную цепь Маркова

$$
x_{1}, x_{2}, \ldots, x_{t}, \ldots,
$$

У которой при любых $\alpha, \alpha_{2}, \ldots, \alpha_{s} \in\{1,2, \ldots, N\}$ и любых $t=1,2, \ldots$

$$
\begin{aligned}
\mathbf{P}\left\{x_{t+s-1}=\alpha \mid x_{t}=\alpha_{1}, x_{t+1}=\alpha_{2}, \ldots, x_{t+s-2}=\alpha_{s-1}\right\} & \\
& =\mathbf{P}\left\{x_{t+s-1}=\alpha_{s} \mid x_{t}=\alpha_{1}, x_{t+r-1}=\alpha_{r}\right\}>0, \quad 1<r<s .
\end{aligned}
$$


Обозначим $P_{\alpha_{s}}^{\left(\alpha_{1}, \alpha_{r}\right)}$ правую часть (2). Последовательность

$$
y_{t}=\left(x_{t}, \ldots, x_{t+s-1}\right), \quad t=1,2 \ldots,
$$

очевидно, образует неразложимую простую однородную цепь Маркова, в которой из любого состояния можно попасть в любое с положительной вероятностью не более, чем за $s$ шагов. Обозначим

$$
\left\{\pi_{\alpha}\right\}, \quad \alpha=\left(\alpha_{1}, \ldots \alpha_{s}\right),
$$

стационарное распределение цепи Маркова (3). Будем предполагать, что

$$
\mathbf{P}\left\{x_{1},=\alpha_{1}, x_{2}=\alpha_{2}, \ldots, x_{s}=\alpha_{s}\right\}=\pi_{\alpha} .
$$

Частоту состояний $\alpha$ в $n$ первых членах последовательности (3) обозначим $\mu_{\alpha}(n)$. Частоту цепочки $i=\left(i_{1}, i_{2}, i_{3}\right)$ в $n$ первых членах последовательности цепочек длины 3

$$
z_{t}=\left(x_{t}, x_{t+r-1}, x_{t+s-1}\right), \quad t=1,2, \ldots,
$$

обозначим $v_{i}(n)$. Распределения частот $\mu_{\alpha}(n), v_{i}(n)$ будем исследовать при $n \rightarrow \infty$ в схеме серий, когда последовательность (1) близка к независимой равновероятной последовательности:

$$
P_{\alpha_{s}}^{\left(\alpha_{1}, \alpha_{r}\right)}(n)=\frac{1}{N}\left(1+\frac{\omega_{\alpha_{s}}^{\left(\alpha_{1}, \alpha_{r}\right)}(n)}{\sqrt{n}}\right)
$$

где

$$
\omega_{\alpha_{s}}^{\left(\alpha_{1}, \alpha_{r}\right)}(n) \rightarrow \omega_{\alpha_{s}}^{\left(\alpha_{1}, \alpha_{r}\right)}, \quad 1<r<s \leqslant N .
$$

Отметим, что при любых $\alpha_{1}, \alpha_{r}$

$$
\sum_{\alpha_{s}=1}^{N} \omega_{\alpha_{s}}^{\left(\alpha_{1}, \alpha_{r}\right)}=0
$$

Частоты $\mu_{\alpha}(n), v_{i}(n)$ удобно представить в виде

$$
\begin{aligned}
\mu_{\alpha}(n) & =\sum_{t=1}^{n} \varepsilon_{\alpha(t)}, \\
\nu_{i}(n) & =\sum_{t=1}^{n} \chi_{i}(t),
\end{aligned}
$$

где

$$
\begin{aligned}
& \varepsilon_{\alpha}(t)=\chi\left(x_{t}=\alpha_{1}, x_{t+1}=\alpha_{2}, \ldots, x_{t+s-1}=\alpha_{s}\right), \\
& \chi_{i}(t)=\chi\left(x_{t}=i_{1}, x_{t+r-1}=i_{2}, x_{t+s-1}=i_{3}\right),
\end{aligned}
$$

$\chi(A)$ - индикатор события $A$.

Лемма 1. Если выполнено условие (5), то стачионарное распределение $\left\{\pi_{\alpha}\right\}$ можно при $n \rightarrow \infty$ представить в виде

$$
\pi_{\alpha}=\frac{1}{N^{s}}\left(1+\frac{\Delta_{\alpha}(n)}{\sqrt{n}}\right), \quad \alpha=\left(\alpha_{1}, \ldots, \alpha_{s}\right),
$$


где величины $\Delta_{\alpha}(n)$ стремятся при $n \rightarrow \infty$ келичинам $\Delta_{\alpha}$, удовлетворяющим равенствам

$$
\Delta_{\alpha_{1} \ldots \alpha_{s}}=\omega_{\alpha_{s}}^{\left(\alpha_{1}, \alpha_{r}\right)}+\frac{1}{N} \sum_{u=1}^{N} \Delta_{u \alpha_{1} \ldots \alpha_{s-1}}, \quad \sum_{\alpha_{1}, \ldots, \alpha_{s}=1}^{N} \Delta_{\alpha_{1} \ldots \alpha_{s}}=0
$$

Доказательство. Доказательство сходимости $\Delta_{\alpha}(n)$ к пределам $\Delta_{\alpha}$ нетрудно получить, если рассматривать вероятности $\pi_{\alpha}=\varphi_{\alpha}(x)$ как функции от $x$, являющиеся решением системы линейных уравнений

$$
\pi_{\alpha}(x)=\sum_{u=1}^{N} \pi_{u \alpha_{1} \ldots \alpha_{s-1}}(x) P_{\alpha_{s}}^{\left(\alpha_{1}, \alpha_{r}\right)}(x), \quad \sum_{\alpha} \pi_{\alpha}(x)=1,
$$

в которой

$$
P_{\alpha_{s}}^{\left(\alpha_{1}, \alpha_{r}\right)}(x)=\frac{1}{N}\left(1+x \omega_{\alpha_{s}}^{\left(\alpha_{1}, \alpha_{r}\right)}\left(1 / x^{\alpha}\right)\right) .
$$

Равенства (9) также можно получить из приведенной системы уравнений.

Из равенств (9) и (6) следует, что

$$
\sum_{v=1}^{N} \Delta_{\alpha_{1} \ldots \alpha_{s-1} v}=\sum_{u=1}^{N} \Delta_{u \alpha_{1} \ldots \alpha_{s-1}} .
$$

Эти равенства будут использованы при вычислении параметров нецентральности $\chi^{2}$-статистик.

Ниже нам потребуется представление, аналогичное (8), для вероятностей

$$
\pi_{i}^{*}=\sum_{\alpha \in G_{i}, i=\left(i_{1}, i_{2}, i_{3}\right)} \pi_{\alpha_{1} \ldots \alpha_{s}}
$$

где

$$
G_{i}=\left\{\alpha: \alpha_{1}=i_{1}, \alpha_{r}=i_{2}, \alpha_{s}=i_{3}\right\}, \quad i=\left(i_{1}, i-2, i_{3}\right) .
$$

Используя лемму 1 , нетрудно получить следующее утверждение.

Лемма 2. Если выполнено условие (5), то

$$
\pi_{i}^{*}=\frac{1}{N^{3}}\left(1+\frac{\Delta_{i}^{*}(n)}{\sqrt{n}}\right), \quad i=\left(i_{1}, i_{2}, i_{3}\right),
$$

где $\Delta_{i}^{*}(n)$ стремятся при $n \rightarrow \infty$ квеличинам

$$
\Delta_{i}^{*}=\frac{1}{N^{s-3}} \sum_{\alpha \in G_{i}} \Delta_{\alpha}
$$

Эти величины удовлетворяют равенствам

$$
\Delta_{i}^{*}=\omega_{i_{3}}^{\left(i_{1}, i_{2}\right)}+\frac{1}{N} \sum_{u=1}^{N} \Delta_{i_{1} i_{2} u}, \quad i=\left(i_{1}, i_{2}, i_{3}\right) .
$$


Скорость сходимости вероятностей перехода $P_{\alpha \beta}(t)$ цепи Маркова (3) к предельным вероятностям $\pi_{\beta}$ оценивается в условиях (5) при всех $n$ и $t$ следующим образом:

$$
\left|P_{\alpha \beta}(t)-\pi_{\beta}\right|<K n^{-(t / s-1) / 2}
$$

где $K$ - некоторая положительная постоянная. Эта оценка непосредственно следует из теоремы на стр. 100 книги Ю. А. Розанова [1], если для коэффициента эргодичности $k(s)$ воспользоваться оценкой

$$
1-k(s)=\frac{1}{2} \sup _{\alpha, \beta} \sum_{\gamma}\left|P_{\alpha \gamma}(s)-P_{\beta \gamma}(s)\right|=O(1 / \sqrt{n})
$$

Приведенные результаты для цепей Маркова (3) будут использованы для получения асимптотических формул моментов частот состояний цепи Маркова (3) и частот цепочек (4).

\section{2. Математические ожидания и ковариации частот}

Из однородности цепи Маркова и равенства (7) следует, что

$$
\begin{aligned}
\mathbf{E} \mu_{\alpha}(n) & =n \pi_{\alpha}, \\
\mathbf{E} \nu_{i}(n) & =n \pi_{i}^{*} .
\end{aligned}
$$

Положим

$$
\begin{aligned}
& \eta_{\alpha}(n)=\frac{\mu_{\alpha}(n)-n / N^{s}}{\sqrt{n / N^{s}}} \\
& \xi_{i}(n)=\frac{\nu_{i}(n)-n / N^{3}}{\sqrt{n / N^{3}}} .
\end{aligned}
$$

Лемма 3. При $n \rightarrow \infty$ в схеме серий (5)

$$
\begin{aligned}
\lim _{n \rightarrow \infty} \mathbf{E} \eta_{\alpha}(n) & =\frac{\Delta_{\alpha}}{N^{s / 2}}, \\
\lim _{n \rightarrow \infty} \mathbf{E} \xi_{i}(n) & =\frac{\Delta_{i}^{*}}{N^{3 / 2}} .
\end{aligned}
$$

Утверждения этой леммы следуют из лемм 1 и 2 и равенств (15).

Будем предполагать, что $2 r \neq s+1$. В случае равенства $\operatorname{cov}\left(\xi_{i}(n), \xi_{j}(n)\right)$ совпадают с ковариациями сплошных цепочек длины 3 . Ковариации величин $\xi(n), \xi_{j}(n)$ представимы в виде

$$
\operatorname{cov}\left(\xi_{i}(n), \xi_{j}(n)\right)=\sum_{l=1}^{6} S_{l}-n N^{3} \pi_{i}^{*} \pi_{j}^{*}
$$


где

$$
\begin{aligned}
S_{l} & =\frac{N^{3}}{n} \sum_{\left(u_{1}, u_{2}\right) \in A_{l}} \mathbf{E}_{\chi_{i}}\left(u_{1}\right) \chi_{j}\left(u_{2}\right), \quad l=1, \ldots, 6, \\
A_{1} & =\left\{\left(u_{1}, u_{2}\right): u_{1}=u_{2}, 1 \leqslant u_{1}, u_{2} \leqslant n\right\}, \\
A_{2} & =\left\{\left(u_{1}, u_{2},:\left|u_{1}-u_{2}\right|=r-1,1 \leqslant u_{1}, u_{2} \leqslant n\right\},\right. \\
A_{3} & =\left\{\left(u_{1}, u_{2}\right):\left|u_{1}-u_{2}\right|=s-1,1 \leqslant u_{1}, u_{2} \leqslant n\right\}, \\
A_{4} & =\left\{\left(u_{1}, u_{2}\right):\left|u_{1}-u_{2}\right|=s-r, 1 \leqslant u_{1}, u_{2} \leqslant n\right\}, \\
A_{5} & =C \cap\left\{\left(u_{1}, u_{2}\right):\left|u_{1}-u_{2}\right| \leqslant s+1,1 \leqslant u_{1}, u_{2} \leqslant n\right\}, \\
A_{6} & =C \cap\left\{\left(u_{1}, u_{2}\right):\left|u_{1}-u_{2}\right| \geqslant s+2,1 \leqslant u_{1}, u_{2} \leqslant n\right\}, \\
C & =\left\{\left(u_{1}, u_{2}\right): 1 \leqslant u_{1} \leqslant n, 1 \leqslant u_{2} \leqslant n\right\} \backslash\left(A_{1} \cup A_{2} \cup A_{3} \cup A_{4}\right) .
\end{aligned}
$$

Отметим, что

$$
\begin{aligned}
\left|A_{1}\right| & =n, \\
\left|A_{2}\right|=\left|A_{3}\right|=\left|A_{4}\right| & =2 n+O(1), \\
|C|=\left|A_{5} \cup A_{6}\right| & =n^{2}-7 n+O(1) .
\end{aligned}
$$

Величины $\chi_{i}\left(u_{1}\right), \chi_{j}\left(u_{2}\right)$ в первых четырех суммах (17) одинаково распределены и, следовательно, эти суммы можно представить в виде

$$
\begin{aligned}
& S_{1}=M_{i j}^{(1)}, \\
& S_{2}=M_{i j}^{(2)}+M_{j i}^{(2)}, \\
& S_{3}=M_{i j}^{(3)}+M_{j i}^{(3)}+O(1 / n), \\
& S_{4}=M_{i j}^{(4)}+M_{j i}^{(4)}+O(1 / n),
\end{aligned}
$$

где

$$
\begin{aligned}
M_{i j}^{(1)} & =N^{3} P\left\{D_{i} \cap D_{j}\right\}, \\
M_{i j}^{(2)} & =N^{3} P\left\{D_{i} \cap\left(x_{r}=j_{1}, x_{2 r-1}=j_{2}, x_{r+s-1}=j_{3}\right)\right\}, \\
M_{i j}^{(3)} & =N^{3} P\left\{D_{i} \cap\left(x_{s}=j_{1}, x_{s+r-1}=j_{2}, x_{2 s-1}=j_{3}\right)\right\}, \\
M_{i j}^{(4)} & =N^{3} P\left\{D_{i} \cap\left(x_{s-r+1}=j_{1}, x_{s}=j_{2}, x_{2 s-r}=j_{3}\right)\right\}, \\
D_{i} & =\left\{x_{1}=i_{1}, x_{r}=i_{2}, x_{s}=i_{3}\right\} .
\end{aligned}
$$

Очевидно, что

$$
M_{i j}^{(1)}=N^{3} \delta_{i j} \pi_{i}^{*},
$$

где $\delta_{i j}-$ символ Кронекера. Отсюда, используя лемму 2, при $n \rightarrow \infty$ получим, что

$$
M_{i j}^{(1)}=\delta_{i j}+O(1 / \sqrt{n}) .
$$

Величину $M_{i j}^{(2)}$ представим в виде

$$
M_{i j}^{(2)}=N^{3} \sum_{\alpha \in G_{i}, \beta \in G_{j}} \pi_{\alpha} P_{\alpha \beta}(r-1) .
$$


Отсюда, используя (5) и (8), нетрудно получить, что

$$
\frac{1}{N^{3}} M_{i j}^{(2)}=\delta_{i_{2} j_{1}} \frac{1}{N^{5}}+O(1 / \sqrt{n})
$$

Аналогично находим, что

$$
\begin{aligned}
& M_{i j}^{(3)}=\delta_{i_{3} j_{1}} \frac{1}{N^{2}}+O(1 / \sqrt{n}) \\
& M_{i j}^{(4)}=\delta_{i_{3} j_{2}} \frac{1}{N^{2}}+O(1 / \sqrt{n})
\end{aligned}
$$

Сумма $S_{5}$ разбивается на конечное число сумм с фиксированной разностью $u_{1}-u_{2}$. Каждая из них оценивается аналогично (21). В сумме, аналогичной (21), фиксируется 6 индексов вместо 5 и поэтому

$$
S_{5}=N^{3} \frac{\left|A_{5}\right|}{n}\left(1 / N^{6}+O(1 / \sqrt{n})\right) .
$$

Отсюда, учитывая (12), получим, что

$$
S_{5}=\frac{\left|A_{5}\right|}{n}\left(N^{3} \pi_{i}^{*} \pi_{j}^{*}+O(1 / \sqrt{n})\right)
$$

Сумму $S_{6}$ представим в виде

$$
S_{6}=\frac{N^{3}}{n} \sum_{\alpha \in G_{i}, \beta \in G_{j}} \sum_{\left(u_{1}, u_{2}\right) \in A_{6}} \pi_{\alpha} P_{\alpha \beta}\left(\left|u_{1}-u_{2}\right|\right) .
$$

Используя это представление и (14), можно показать, что

$$
S_{6}=\frac{\left|A_{6}\right|}{n}\left(N^{3} \pi_{i}^{*} \pi_{j}^{*}+O(1 / \sqrt{n})\right)
$$

Из равенств (17), (18), (20), (22)-(25) находим асимптотическую формулу для ковариаций (17). Полученные результаты приведем в виде следующей теоремы.

Теорема 1. Если $2 r \neq s+1$, то при $n \rightarrow \infty$ в схеме серий (5)

$$
\begin{aligned}
& \lim _{n \rightarrow \infty} \mathbf{E} \xi_{i}(n)=\omega_{i_{3}}^{\left(i_{1}, i_{2}\right)} / \sqrt{N^{3}}, \\
& \lim _{n \rightarrow \infty} \operatorname{cov}\left(\xi_{i}(n), \xi_{j}(n)\right)=b_{i j},
\end{aligned}
$$

гेe

$$
b_{i j}=\delta_{i j}+\frac{1}{N^{2}}\left(\delta_{i_{2} j_{1}}+\delta_{i_{3} j_{1}}+\delta_{i_{3} j_{2}}+\delta_{j_{2} i_{1}}+\delta_{j_{3} i_{1}}+\delta_{j_{3} i_{2}}\right)-\frac{7}{N^{3}}
$$




\section{3. Предельные распределения}

Частоты $\mu_{\alpha}(n)$ состояний цепи Маркова (3) при $n \rightarrow \infty$ в схеме серий (5) асимптотически нормальны. Это утверждение нетрудно получить, если воспользоваться теоремой 1 из [6]. Отсюда следует асимптотическая нормальность $\nu_{i}(n)$, являющихся суммами частот $\mu_{\alpha}(n)$.

Положим

$$
\begin{aligned}
\tilde{\mu}_{\tilde{\alpha}}(n) & \left.=\sum_{\alpha_{s}=1}^{N} \mu_{\alpha}(n)\right), \\
\tilde{\alpha} & =\left(\alpha_{1}, \ldots, \alpha_{s-1}\right), \quad \alpha=\left(\alpha_{1}, \ldots, \ldots, \alpha_{s}\right), \\
\tilde{v}_{\left(i_{1}, i_{2}\right)}(n) & =\sum_{i_{3}=1}^{N} \nu_{i_{1} i_{2} i_{3}}(n) ; \\
\tilde{\eta}_{\tilde{\alpha}}(n) & =\frac{\tilde{\mu}_{\tilde{\alpha}}(n)-n / N^{s-1}}{\sqrt{n / N^{s-1}}}, \\
\tilde{\xi}_{\left(i_{1}, i_{2}\right)}(n) & =\frac{\tilde{\nu}_{\left(i_{1}, i_{2}\right)}(n)-n / N^{2}}{\sqrt{n / N^{2}}} .
\end{aligned}
$$

Нормированные величины (27) можно выразить через величины (16) следующим образом:

$$
\begin{aligned}
\tilde{\xi}_{\left(i_{1}, i_{2}\right)}(n) & =\frac{1}{\sqrt{N}} \sum_{i_{3}=1}^{N} \xi_{i_{1} i_{2} i_{3}}(n), \\
\tilde{\eta}_{\tilde{\alpha}}(n) & =\frac{1}{\sqrt{N}} \sum_{\alpha_{s}=1}^{N} \mu_{\alpha}(n) .
\end{aligned}
$$

Статистики типа $\chi^{2}$, построенные по частотам несплошных и сплошных цепочек, огределим следующим образом:

$$
\begin{aligned}
& X(n)=\sum_{i_{1}, i_{2}, i_{3},=1}^{N} \xi_{i}^{2}(n)-\sum_{i_{1}, i_{2}=1}^{N} \tilde{\xi}_{\left(i_{1}, i_{2}\right)}^{2}(n), \\
& Y(n)=\sum_{\alpha_{1}, \ldots, \alpha_{s}=1}^{N} \eta_{\alpha}^{2}(n)-\sum_{\alpha_{1}, \ldots, \alpha_{s-1}=1} \tilde{\eta}_{\tilde{\alpha}}^{2}(n) .
\end{aligned}
$$

Сходимость при $n \rightarrow \infty$ распределения статистики (30) к нецентральному $\chi^{2}$-распределению хорошо известна. Это утверждение можно получить из результатов работ [2] и [3]. Выразим параметр нецентральности $a_{Y}^{2}$ предельного распределения $Y(n)$ через параметры рассматриваемой цепи Маркова. По второй формуле (28), используя лемму 3 , при $n \rightarrow \infty$ получим, что

$$
\mathbf{E} \tilde{\eta}_{\tilde{\alpha}}(n)=\frac{1}{\sqrt{N}} \sum_{\alpha_{s}=1}^{N} \mathbf{E} \eta_{\alpha}(n) \rightarrow \frac{1}{N^{(s+1) / 2}} \sum_{\alpha_{s}=1}^{N} \Delta_{\alpha}
$$


Отсюда, используя лемму 3 и равенство

$$
a_{Y}^{2}=\lim _{n \rightarrow \infty}\left(\sum_{\alpha_{1}, \ldots, \alpha_{s}=1}^{N}\left(\mathbf{E} \eta_{\alpha}(n)\right)^{2}-\sum_{\alpha_{1}, \ldots, \alpha_{s-1}=1}\left(\mathbf{E} \tilde{\eta}_{\tilde{\alpha}}(n)\right)^{2}\right),
$$

получим равенство

$$
N^{s} a_{Y}^{2}=\sum_{\alpha_{1}, \ldots, \alpha_{s}=1}^{N} \Delta_{\alpha}^{2}-\frac{1}{N} \sum_{\alpha_{1} \ldots, \alpha_{s-1}=1}^{N}\left(\sum_{\alpha_{s}}^{N} \Delta_{\alpha}\right)^{2} .
$$

Из равенства (9) следует, что

$$
\Delta_{\alpha}^{2}=\left(\omega_{\alpha_{s}}^{\left(\alpha_{1}, \alpha_{r}\right)}\right)^{2}+\frac{2}{N} \omega_{\alpha_{1}}^{\left(\alpha_{1}, \alpha_{r}\right)} \sum_{u=1}^{N} \Delta_{u \alpha_{1} \ldots \alpha_{s-1}}+\frac{1}{N^{2}} \sum_{u, v=1}^{N} \Delta_{u \alpha_{1} \ldots \alpha_{s-1}} \Delta_{v \alpha_{1} \ldots \alpha_{s-1}} .
$$

Отсюда и из равенств (6), (10) получаем равенство

$$
\sum_{\alpha_{1}, \ldots, \alpha_{s}=1}^{N} \Delta_{\alpha}^{2}=N^{s-3} \sum_{i_{1}, i_{2}, i_{3}=1}^{N}\left(\omega_{i_{3}}^{\left(i_{1}, i_{2}\right)}\right)^{2}+\frac{1}{N} \sum_{\alpha_{1}, \ldots, \alpha_{s}=1}^{N}\left(\sum_{u=1}^{N} \Delta_{\alpha_{1} \ldots \alpha_{s-1} u}\right)^{2} .
$$

Подставив это выражение в (31), найдем, что

$$
a_{Y}^{2}=a^{2}
$$

где

$$
a^{2}=\frac{1}{N^{3}} \sum_{\alpha_{1}, \alpha_{r}, \alpha_{s}=1}\left(\omega_{\alpha_{s}}^{\left(\alpha_{1}, \alpha_{r}\right)}\right)^{2}
$$

Приведем этот частный случай общего утверждения о сходимости к $\chi^{2}$ - распределению в виде следуюшей теоремы.

Теорема 2. При $n \rightarrow \infty$ в схеме серий (5) распределение статистики $Y(n)$ сходится $\kappa$ нечентральному $\chi^{2}$-распределению $c N^{s}-N^{s-1}$ степенями свободы и параметром нецентральности $a^{2}$, определенным равенством (32).

Статистика $X(n)$ является квадратичной формой от нормированных частот $\left\{\xi_{i}(n)\right\}$. Обозначим $A=\left(A_{i j}\right)$ матрицу коэффициентов этой квадратичной формы. Предельную матрицу ковариаций $b_{i j}$, определенных в теореме 1 , обозначим $B$. Для того чтобы статистика $X(n)$ имела в пределе $\chi^{2}$ - распределение (центральное или нецентральное) достаточно (см. [4]), чтобы выполнялось условие $A B A=A$.

Это условие было проверено в [5] для случая равновероятной независимой последовательности. В рассматриваемой схеме серий (5) предельная ковариационная матрица совпала с ковариационной матрицей для независимой равновероятной последовательности и, следовательно, это условие проверено. Параметр нецентральности $a_{X}^{2}$ предельного распределения определяется равенством

$$
a_{X}^{2}=\lim _{n \rightarrow \infty}\left(\sum_{i_{1}, i_{2} i_{3}=1}^{N}\left(\mathbf{E} \xi_{i}(n)\right)^{2}-\sum_{\left(i_{1}, i_{2}\right)}^{N}\left(\mathbf{E} \tilde{\xi}_{\left(i_{1}, i_{2}\right)}(n)\right)^{2}\right) .
$$

Отсюда, используя леммы 2,3 и равенство (28), нетрудно получить, что $a_{X}^{2}=a^{2}$.

Таким образом, получено следующее утверждение. 
Теорема 3. При $n \rightarrow \infty$ в схеме серий (5) распределение статистики $X(n)$ сходится к нецентральному $\chi^{2}$-распределению $c N^{3}-N^{2}$ степенями свободы и параметром нечентральности $a^{2}$, определенным формулой (31).

Статистики $X(n)$ и $Y(n)$ могут быть использованы для различения основной гипотезы $H_{0}$ (последовательность (1) является независимой и равновероятной) и конкурирующей $H_{1}$, определенной равенством (2). Если вероятности переходов в левой части равенства (2) зависят от $v-1$ знаков, $v>3$, то потребуется статистика, зависящая от частот несплошных цепочек длины $v$.

Теорема 3 в случае $v>3$ очевидным образом формулируется, и ее доказательство существенно не отличается от доказательства, проведенного для частот цепочек длины 3.

\section{4. Статистические критерии}

Определим $X$-критерий и $Y$-критерий. Гипотеза $H_{1}$ по $X$-критерию со статистикой $X(n)$ принимается, если $X(n)>C_{X}$, где $C_{X}$ - некоторая положительная постоянная. Такое же условие для $Y$-критерия со статистикой $Y(n)$ : гипотеза $H_{1}$ принимается, если $Y(n)>C_{Y}$, $C_{Y}>0$.

Сравним эти два критерия в случае больших $N$, когда можно воспользоваться нормальных приближением предельных распределений. Обозначим $X$ и $Y$ нормально распределенные величины, у которых первые два момента при гипотезах $H_{0}$ и $H_{1}$ определяются равенствами

$$
\begin{array}{ll}
\mathbf{E}_{H_{0}} X=N^{3}-N^{2}, & \mathbf{D}_{H_{0}} X=2\left(N^{3}-N^{2}\right), \\
\mathbf{E}_{H_{1}} X=N^{3}-N^{2}+a^{2}, & \mathbf{D}_{H_{1}} X=2\left(N^{3}-N^{2}\right)+4 a^{2}, \\
\mathbf{E}_{H_{0}} Y=N^{s}-N^{s-1}, & \mathbf{D}_{H_{0}} Y=2\left(N^{s}-N^{s-1}\right), \\
\mathbf{E}_{H_{1}} Y=N^{s}-N^{s-1}+a^{2}, & \mathbf{D}_{H_{1}} Y=2\left(N^{s}-N^{s-1}\right)+4 a^{2} .
\end{array}
$$

Если задать для обоих критериев одинаковую вероятность ошибки первого рода $\alpha$, то их вероятности ошибок 2-го рода $\beta_{X}, \beta_{Y}$ определяются равенствами

$$
\begin{aligned}
& u_{\beta_{X}}=\frac{a^{2}}{\sqrt{2\left(N^{3}-N^{2}\right)+4 a^{2}}}-u_{\alpha}\left(1+\frac{2 a^{2}}{N^{3}-N^{2}}\right)^{-1 / 2}, \\
& u_{\beta_{Y}}=\frac{a^{2}}{\sqrt{2\left(N^{s}-N^{s-1}\right)+4 a^{2}}}-u_{\alpha}\left(1+\frac{2 a^{2}}{N^{3}-N^{2}}\right)^{-1 / 2},
\end{aligned}
$$

где $u_{\gamma}$ - квантиль нормального распределения:

$$
\frac{1}{\sqrt{2 \pi}} \int_{u_{\gamma}}^{\infty} e^{-x^{2} / 2} d x=\gamma
$$

При больших $N$

$$
\frac{u_{\beta_{X}}}{u_{\beta_{Y}}} \sim \sqrt{\left(N^{s}-N^{s-1}\right) /\left(N^{3}-N^{2}\right)} \sim N^{s / 6}
$$

и, следовательно, $X$-критерий является более мощным по сравнению с $Y$-критерием.

Статистика $X(n)$ для произвольных несплошных цепочек в работе [5] была определена по аналогии с $\chi^{2}$-статистиками $Y(n)$, предложенными в [2]. Ранг ковариационной 
матрицы частот сплошных $s$-цепочек совпадает с числом степеней свободы предельного $\chi^{2}$-распределения. Ранг ковариационной матрицы частот несплошных цепочек не совпадает с числом степеней свободы предельного распределения. Например, при $N=2$ ранг ковариационной матрицы равен 5 , а число степеней свободы $N^{3}-N^{2}=4$. Таким образом, вместо $X(n)$ можно использовать более сложные квадратичные формы частот, не приводящее к потере степеней свободы и мощности критериев.

Отметим, что для вычисления квадратичных форм $X(n), Y(n)$ от частот цепочек нужно знать только распределение при гипотезе $H_{0}$. Для вычисления характеристик этих критериев нужны еще значения параметров $a^{2}, N, s$. Таким образом, эти кригтерии могут быть использованы в случае, когда гипотеза $H_{1}$ является сложной (любые $\omega_{\alpha_{s}}^{\left(\alpha_{1}, \alpha_{r}\right)}$ с фиксированным значением $a^{2}$ ).

Статистика критерия Неймана-Пирсона, когда обе гипотезы $H_{0}$ и $H_{1}$ простые, зависит от всех $\omega_{\alpha_{s}}^{\left(\alpha_{1}, \alpha_{r}\right)}$. Функщия правдоподобия $L\left(x_{1}, \ldots, x_{n+s-1}\right)$ при гипотезе $H_{1}$ имеет вид

$$
L\left(x_{1}, \ldots, x_{n+s-1}\right)=\prod_{t=1}^{N} p_{x_{t+s-1}}^{\left(x_{t}, x_{t+r-1}\right)} .
$$

Отсюда,

$$
\begin{aligned}
\ln L\left(x_{1}, \ldots, x_{n+s-1}\right) & =\sum_{t=1}^{n} \ln p_{x_{t+s-1}}^{\left(x_{t}, x_{t+r-1}\right)} \\
& =\sum_{i_{1}, i_{2}, i_{3}=1}^{N} v_{\left(i_{1} i_{2} i_{3}\right)}(n) \ln p_{i_{3}}^{\left(i_{1} i_{2}\right)} .
\end{aligned}
$$

и, следовательно, статистику $\tilde{Z}_{n}$ критерия Неймана-Пирсона, используя (5), можно записать в виде

$$
\tilde{Z}_{n}=\sum_{i_{1}, i_{2}, i_{3}=1}^{N} v_{i}(n) \ln \left(1+\omega_{i_{3}}^{\left(i_{1}, i_{2}\right)} / \sqrt{n}\right) .
$$

Приведем характеристики критерия, близкого к критерию Неймана-Пирсона, со статистикой

$$
Z(n)=\sum_{i_{1}, i_{2}, i_{3}=1}^{N} \omega_{i_{3}}^{\left(i_{1}, i_{2}\right)} \xi_{i}(n) .
$$

При $n \rightarrow \infty$ в схеме серий (5) эта статистика асимптотически нормальна. Обозначим $Z$ случайную величину, распределение которой совпадает с предельным распределением для $Z(n)$.

Используя теорему 1 , нетрудно получить первые два момента при гипотезах $H_{0}$ и $H_{1}$ :

$$
\mathbf{E}_{H_{0}} Z=0, \quad \mathbf{D}_{H_{0}} Z=\mathbf{D}_{H_{1}} Z=N^{3} a^{2}, \quad \mathbf{E}_{H_{1}} Z=a^{2} N^{3 / 2} .
$$

Вероятности ошибок 1-го и 2-го рода, $\alpha$ и $\beta_{Z}$, критерия со статистикой $Z$ связаны равенством

$$
u_{\beta_{Z}}=a-u_{\alpha}
$$




\section{Список литературы}

1. Розанов Ю А., Случайные прочессы. Наука, Москва, 1979.

2. Степанов В. Е., Некоторые статистические критерии для цепей Маркова. Теория вероятностей и ее применения (1957) 2, №1, 143-144.

3. Селиванов Б. И., Чистяков В. П., Последовательный $\chi^{2}$-критерий, построенный по $s$-цепочкам состояний цепи Маркова. Дискретная математика (1997) 9, №4, 127-136.

4. Dik I. I., Gunst M. C. M., The distribution of general guadratic form in normal variables. Stat. Neerlandica (1985) 39, №1, 14-26.

5. Чистяков В. П., Статистики хи-квадрат, построенные по частотам несплошных цепочек исходов независимых испытаний. Дискретная математика (2001) 13, №4, 52-59.

6. Тихомирова М. И., Чистяков В. П., Проверка серий и интервалов. Обозрение прикладной и промышиенной математики (1995) 2, №6, 980-993.

Статья поступила 29.01.2003. 